\title{
Thermal instability of a rotating saturated porous medium heated from below and submitted to rotation
}

\author{
Th. Desaive ${ }^{1, a}$, M. Hennenberg ${ }^{2}$, and G. Lebon ${ }^{1}$ \\ 1 Institute of Physics, Department of Astrophysics and Geophysics, University of Liège, Belgium \\ 2 Microgravity Research Center, Fac. des Sciences Appliquées, Université Libre de Bruxelles, CP 165/62, \\ Av. F.D. Roosevelt, 1050, Bruxelles, Belgium
}

Received 24 June 2002 / Received in final form 11 September 2002

Published online 31 October 2002 - (c) EDP Sciences, Società Italiana di Fisica, Springer-Verlag 2002

\begin{abstract}
In this work, we study the problem of onset of thermal convection in a rotating saturated porous medium heated from below. The effect of rotation is restricted to the Coriolis force, neglecting thus the centrifugal effects, the porous medium is described by Brinkman's model. The linear eigenvalue problem is solved by means of a modified Galerkin method. The behavior of the critical temperature gradient is discussed in terms of various parameters of the system for both stationary and overstable convections. Finally a weakly nonlinear analysis is provided to derive amplitude equations and to study the onset of Küppers-Lortz instability.
\end{abstract}

PACS. 47.20.Bp Buoyancy-driven instability - 44.25. $+\mathrm{f}$ Natural convection -47.27 .Te Convection and heat transfer

\section{Introduction}

The study of fluid convection in a rotating porous medium is not only a subject of fundamental importance but also of practical interest; it is present in a large number of applications, such as centrifugal filtration processes, food engineering and geophysics. A good review of flow interaction and heat transfer in porous media is found in [1] and a detailed analysis of the effect of rotation in Vadasz [2]. The influence of Coriolis effect on gravity-driven convection in porous media was first investigated by Friedrichs [3] who used the Brinkman model [4] to describe the linear stability while Palm and Tyvand [5] employed a classical Darcy model. Vadasz [6] based its analysis on an extended Darcy model with a time-derivative term in the momentum equation allowing for overstability. He found that, in contrast with the same problem in pure fluid [7], overstable convection in rotating porous media is not restricted to a particular range of Prandtl numbers. By means of a weakly nonlinear analysis, he also showed that rotation may have a retarding effect on convective heat transfer.

Our first aim is to develop a linear stability analysis of a rotating porous layer described by Brinkman's model and to detail the influence of several dimensionless parameters on the critical values. Second, a weakly nonlinear regime based on a projection method is investigated and amplitude equations are derived. These amplitude equations are used to study the onset of the Küppers-Lortz

\footnotetext{
a e-mail: tdesaive@ulg.ac.be
}

instability and to determine the angle as well as the corresponding Taylor number at which the Küppers-Lortz instability first manifests itself when the angular velocity is increased.

\section{Mathematical formulation}

We consider a homogeneous porous medium of thickness $d$ and infinite horizontal extent saturated by an incompressible fluid. The system is heated from below in the gravity field and is rotating with a uniform angular velocity $\boldsymbol{\Omega}$ around a vertical axis $\mathbf{e}_{z}$ (Fig. 1). We take for granted the Boussinesq approximation and assume that the saturating fluid is Newtonian with density given by

$$
\rho=\rho_{0}\left[1-\alpha_{T}\left(T-T_{0}\right)\right],
$$

where $\rho_{0}$ is the density of the liquid at temperature $T_{0}$ and $\alpha_{T}$ the constant coefficient of volumic expansion.

Although Darcy's law is widely used to describe porous media, we prefer to select the Brinkman model that accounts for friction caused by macroscopic shear. It is known that this effect seriously affects the flow especially in sparsely packed porous media. This amounts to considering the saturated porous matrix as a specific fluid with an effective viscosity $\mu_{e}$ and subject to an additional external body force, namely, the Darcy force. For distances, not too far from the axis of rotation, one can neglect the centrifugal effects $[8,9]$, hence limiting the effect of rotation to the Coriolis force. In a reference frame linked 


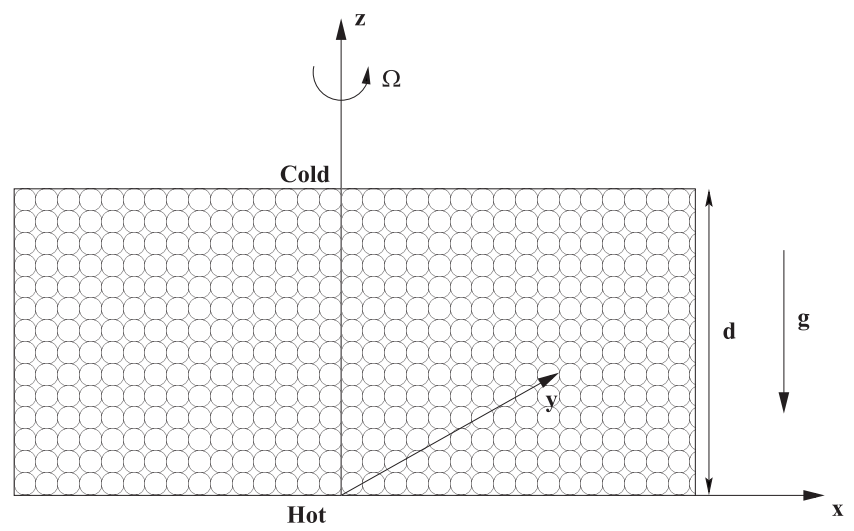

Fig. 1. Geometrical configuration.

to the rotating porous matrix, the continuity, momentum and energy equation read therefore as

$$
\boldsymbol{\nabla} \cdot \mathbf{u}=0
$$

$$
\begin{aligned}
\frac{\rho_{0}}{\phi}\left(\partial_{t} \mathbf{u}+\frac{1}{\phi} \mathbf{u} \cdot \nabla \mathbf{u}+2 \boldsymbol{\Omega} \times \mathbf{u}\right) & =-\nabla p+\mu_{e} \nabla^{2} \mathbf{u}-\frac{\mu_{l}}{K} \mathbf{u} \\
& -\rho_{0} g\left[1-\alpha_{T}\left(T-T_{0}\right)\right] \mathbf{e}_{z},
\end{aligned}
$$

$$
\partial_{t} T+\frac{\left(\rho_{0} c\right)_{l}}{\left(\rho_{0} c\right)_{p}}(\mathbf{u} \cdot \nabla T)=\frac{k_{p}}{\left(\rho_{0} c\right)_{p}} \nabla^{2} T,
$$

wherein $\phi$ designates the porosity, $K$ the permeability, $\boldsymbol{\Omega}$ the angular velocity, $\mathbf{u}=(u, v, w)$ the seepage velocity (i.e. the average of the fluid velocity over a volume element of the porous medium), $p$ the pressure, $T$ the temperature, $k_{p}$ is the mean thermal conductivity in the porous layer. For any physical property, one has ()$_{p}=(1-\phi)()_{s}+$ $\phi()_{l}$ where subscript $s$ denotes a property of the solid matrix while subscript $l$ refers to the liquid.

\section{Linear stability analysis}

\subsection{Small perturbation equations and relevant boundary conditions}

To study the linear stability of the system, the analysis is restricted to infinitesimally small perturbations. The governing equations are linearized with respect to the reference state, namely, a motionless fluid with a steady temperature difference $\Delta T$ between the bottom and top surfaces. For convenience, the variables are written in dimensionless form and the following scaling units are used: $d$ for the length, $\Delta T$ for the temperature, $d^{2} / \kappa_{p}=$ $d^{2}\left(\rho_{o} c\right)_{l} / k_{p}$ for the time and $\kappa_{p} / d$ for the velocity. After some straightforward calculations, one obtains the following dimensionless linearized equations for the perturbed variables namely the vertical component of the velocity $w$, the vertical component of the vorticity $\zeta\left(=(\boldsymbol{\nabla} \times \mathbf{u}) \cdot \mathbf{e}_{z}\right)$ and the temperature $T$ :

$$
\begin{aligned}
\chi^{-1} \partial_{t} \nabla^{2} w= & R a \nabla_{h}^{2} T+\Lambda D a \nabla^{4} w \\
& -\nabla^{2} w-\sqrt{T a} \partial_{z} \zeta, \\
\chi^{-1} \partial_{t} \zeta= & \Lambda D a \nabla^{2} \zeta-\zeta+\sqrt{T a} \partial_{z} w, \\
S^{-1} \partial_{t} T= & \nabla^{2} T+w,
\end{aligned}
$$

all the symbols refer to dimensionless perturbed quantities; we have kept for simplicity the same notation for the perturbed and overall fields. Six dimensionless parameters have been introduced in the above equations, namely

$$
\begin{aligned}
R a & =\frac{\alpha_{T} g \rho_{0} \Delta T d K}{\mu_{l} \kappa_{p}}, \text { the porous Rayleigh number, } \\
\chi & =\frac{\mu_{l} d^{2} \phi}{\rho_{0} \kappa_{p} K}, \text { the filtration Prandtl number, } \\
D a & =\frac{K}{d^{2}}, \text { the Darcy number, } \\
\Lambda & =\frac{\mu_{e}}{\mu_{l}}, \text { the dynamic viscosities ratio, } \\
T a & =\left(\frac{2 \Omega \rho_{0} K}{\mu_{l} \phi}\right)^{2}, \text { the porous Taylor number, } \\
S & =\frac{(\rho c)_{l}}{(\rho c)_{p}}, \text { the heat capacities ratio. }
\end{aligned}
$$

To solve the set (5-7), we need eight boundary conditions which are given below.

At the lower wall $(z=0)$ : The boundary is assumed rigid and perfectly heat conducting, so that

$$
\begin{aligned}
w & =0, \\
\partial_{z} w & =0, \\
\zeta & =0, \\
T & =0 .
\end{aligned}
$$

At the upper surface $(z=1)$ : The upper wall is also considered to be rigid with

$$
\begin{aligned}
w & =0, \\
\partial_{z} w & =0, \\
\zeta & =0,
\end{aligned}
$$

while heat transfer is assumed to be governed by Newton's cooling law

$$
\partial_{z} T+\frac{B i}{1-B i} T=0,
$$

wherein $B i=\frac{B}{1+B}$ is the modified Biot number running from 0 (adiabatic wall) to 1 (perfectly heat conducting wall) while $B$ is the classical Biot number.

\subsection{Normal mode decomposition}

According to the normal mode technique, one seeks solutions for the three unknown fields of the form

$$
\left(\begin{array}{c}
w \\
\zeta \\
T
\end{array}\right)=\left(\begin{array}{c}
W(z) \\
Z(z) \\
\Theta(z)
\end{array}\right) \exp \left[\mathrm{i}\left(a_{x} x+a_{y} y\right)+s t\right]
$$


where the amplitudes $W(z), Z(z)$ and $\Theta(z)$ describe the unknown variation with respect to $z$ of the vertical velocity, the vertical vorticity and the temperature respectively, $a_{x}$ and $a_{y}$ are the dimensionless wave numbers in the $x$ and $y$ directions respectively, finally, $s$ is the complex growth rate of the disturbances.

Introducing expression (16) in equations (5-7) leads to

$$
\begin{aligned}
\chi^{-1} \phi^{-1} s\left(D^{2}-a^{2}\right) W= & \Lambda D a\left(D^{2}-a^{2}\right)^{2} W \\
& -\left(D^{2}-a^{2}\right) W-a^{2} R a \Theta \\
& -\sqrt{T a} D Z \\
\chi^{-1} \phi^{-1} s Z= & \Lambda D a\left(D^{2}-a^{2}\right) Z-Z \\
& +\sqrt{T a} D W \\
S^{-1} s T= & \left(D^{2}-a^{2}\right) \Theta+W
\end{aligned}
$$

where $D$ stands for $d / d z$ and $a=\sqrt{a_{x}^{2}+a_{y}^{2}}$ is an overall horizontal wave number. The above three equations give rise two a 8 th order system with the following associated boundary conditions. At $z=0$, one has

$$
\begin{aligned}
W & =0, \\
D W & =0, \\
Z & =0, \\
\Theta & =0 .
\end{aligned}
$$

At $z=1$ :

$$
\begin{aligned}
W & =0, \\
D W & =0, \\
Z & =0, \\
D \Theta+\frac{B i}{1-B i} \Theta & =0 .
\end{aligned}
$$

The parameters $\chi$ and $S$ are still present in the set (17-19) because oscillatory convection is allowed; the principle of exchange of stability is not supposed to hold, i.e. $s$ is not set equal to zero at marginal stability. It is worth noting that for each value of the horizontal wave number $a$ (which can take all directions and moduli in the case of a system of infinite horizontal extent) the linear eigenproblem (17-19) admits an infinite set of eigenvalues that will be distinguished by means of the subscript $q$ in our nonlinear analysis, with corresponding vertical eigenfunctions $W_{q}(z), Z_{q}(z)$ and $\theta_{q}(z)$ for the vertical velocity, vertical vorticity and temperature perturbations. The maximum value of the growth rate is zero and corresponds to a wave number equal to the critical value $a_{c}$. The in$\operatorname{dex} q$ runs from 1 to infinity and the negative growth rates are assumed to be ordered in such a way that the real part of the growth rate $s_{q}$ decreases with $q$ (for $a=a_{c}$, one has $\left.s_{1}=0\right)$. The eigenmodes are normalized in such a way that the maximum value of the modulus of $\theta_{q}(z)$ is equal to 1 . The linear eigenproblem is solved by means of a modified Galerkin method based on a Chebyshev polynomial expansion of the eigenfunctions [10].

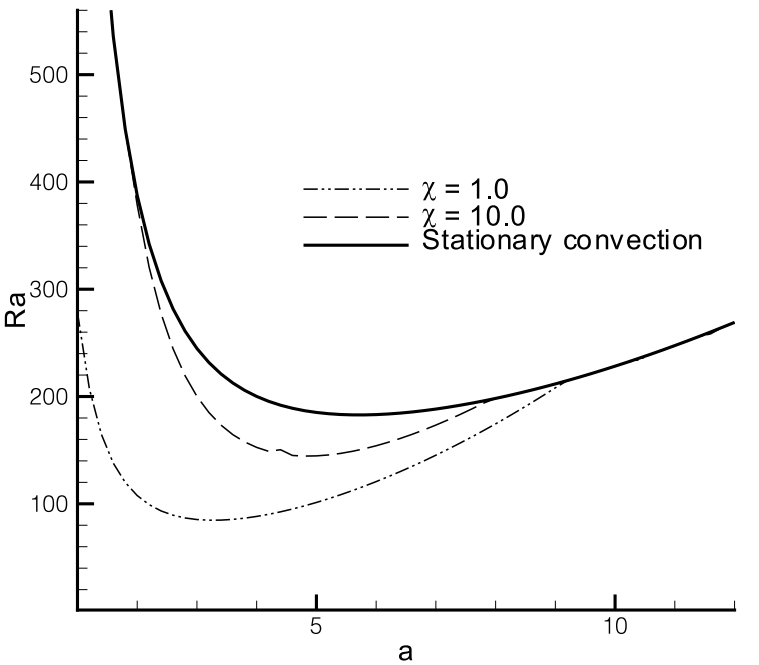

Fig. 2. Neutral stability curves for $T a=10.0, D a=10^{-5}$ and $S=1.0$. Dashed curves represent overstable convection and the solid curve represents the stationary convection.

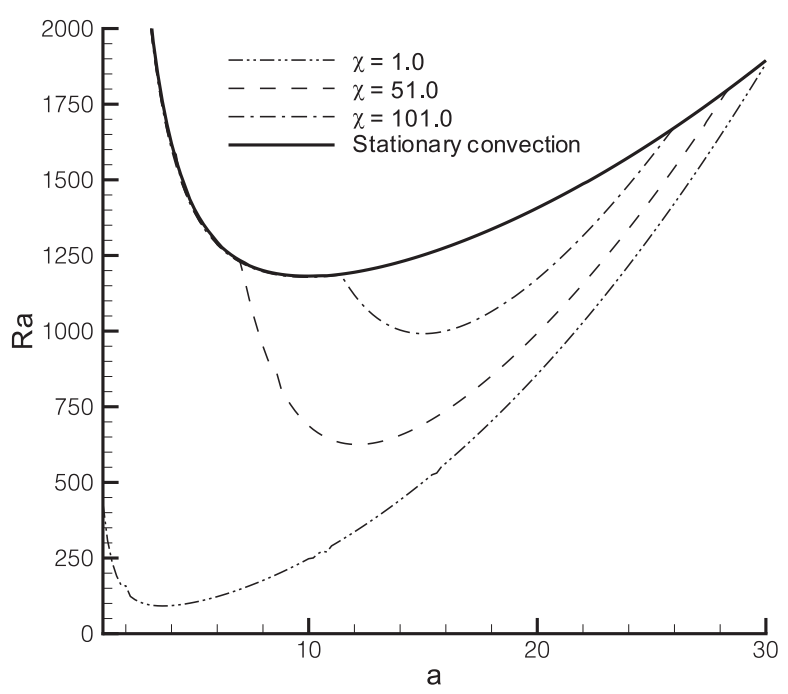

Fig. 3. Neutral stability curves for $T a=100.0, D a=10^{-5}$ and $S=1.0$. Dashed curves represent overstable convection and the solid curve represents stationary convection.

\subsection{Results for Brinkman's model}

Figures 2 and 3 represent the neutral stability curves expressing $R a$ in terms of the wave number $a$ for $T a=10.0$ and $T a=100.0$. These results are qualitatively similar to the stability curves obtained by Vadasz [6] with an extended Darcy model. The neutral curves may exhibit one or two minima that is either overstable or stationary. In the particular case where both stationary and overstable convection occur for the same value of the critical Rayleigh number, one gets a codimension- 2 point. It appears clearly that regions where overstable convection occurs depend on the values of $T a$ and $\chi$.

Our Brinkman's model allows for analyzing the influence of $D a$ on the neutral stability curves. Figures 4 and 5 represent this influence for two different values of $T a$. For 


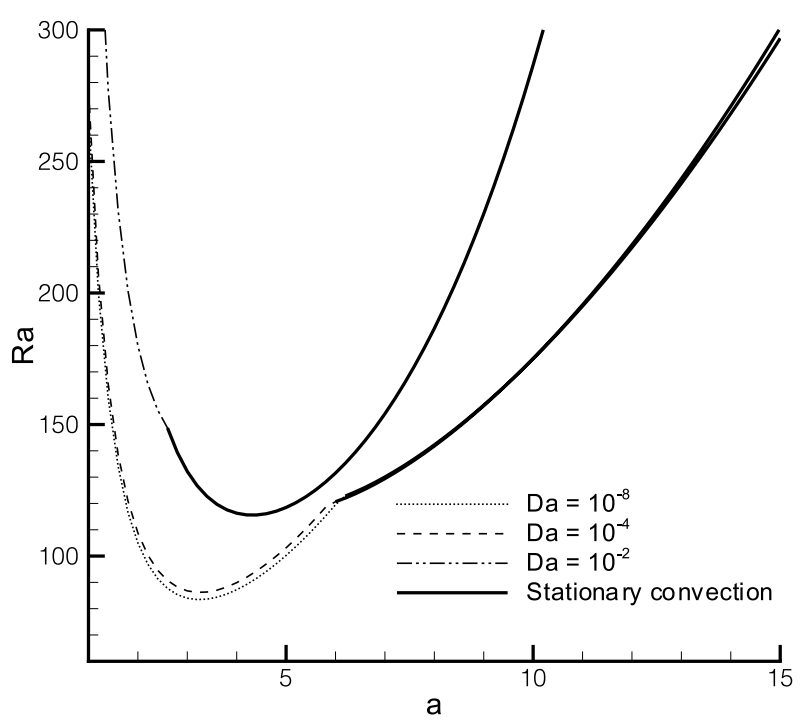

Fig. 4. Influence of the Darcy number for $T a=5.0, \chi=1.0$ and $S=1.0$. Dashed curves represent overstable convection and the solid curve represents stationary convection.

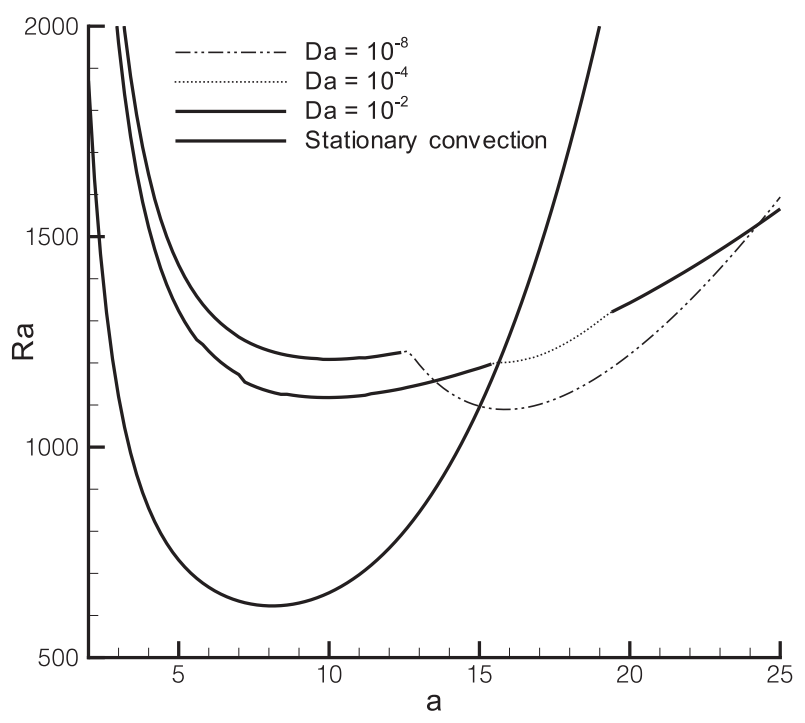

Fig. 5. Influence of the Darcy number for $T a=100.0, \chi=$ 120.0 and $S=1.0$. Dashed curves represent overstable convection and the solid curve represents the stationary convection.

very low values of $D a$, the critical value is stationary or overstable and one recovers (see Fig. 6) the analytical results obtained by Vadasz [6] with Darcy model, namely

$$
\begin{aligned}
& R a_{c}^{\text {over }}=\frac{2}{a^{2}}\left[\left(\pi^{2}+a^{2}\right)\left(\pi^{2}+a^{2}+\chi\right)+\frac{\chi^{2} T a \pi^{2}}{\pi^{2}+a^{2}+\chi}\right], \\
& R a_{c}^{\text {stat }}=\frac{\left(\pi^{2}+a^{2}\right)^{2}}{a^{2}}+T a \frac{\left(\pi^{2}+a^{2}\right)}{a^{2}} \pi^{2},
\end{aligned}
$$

where $R a_{c}^{\text {over }}$ and $R a_{c}^{\text {stat }}$ denote the overstable and stationary porous Rayleigh numbers respectively. When $D a$ is increased, one observes that the critical convection is always stationary, as could have been expected because the

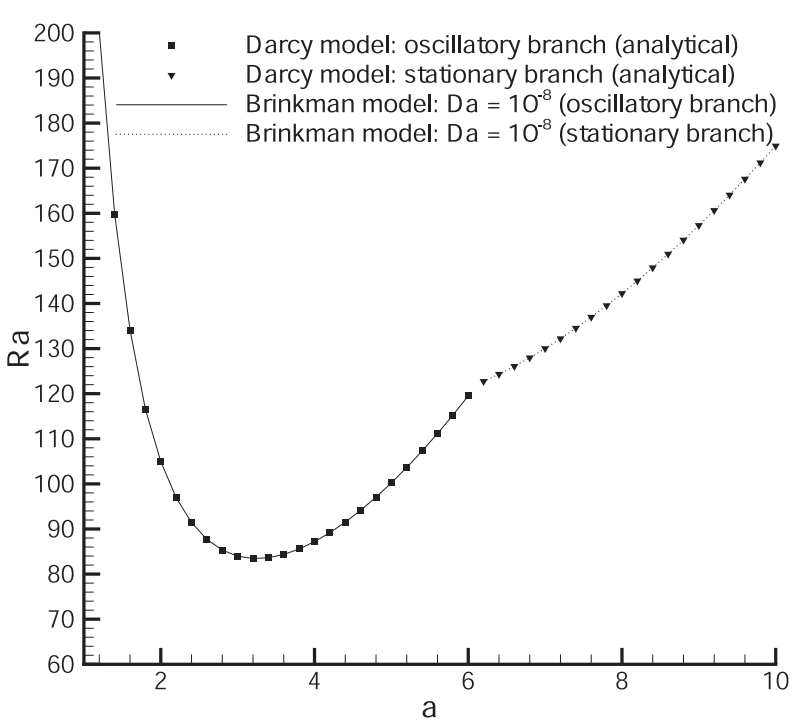

Fig. 6. Stability curves for Brinkman model and analytical results from Darcy model $(T a=5.0, \chi=1.0$ and $S=1.0)$.

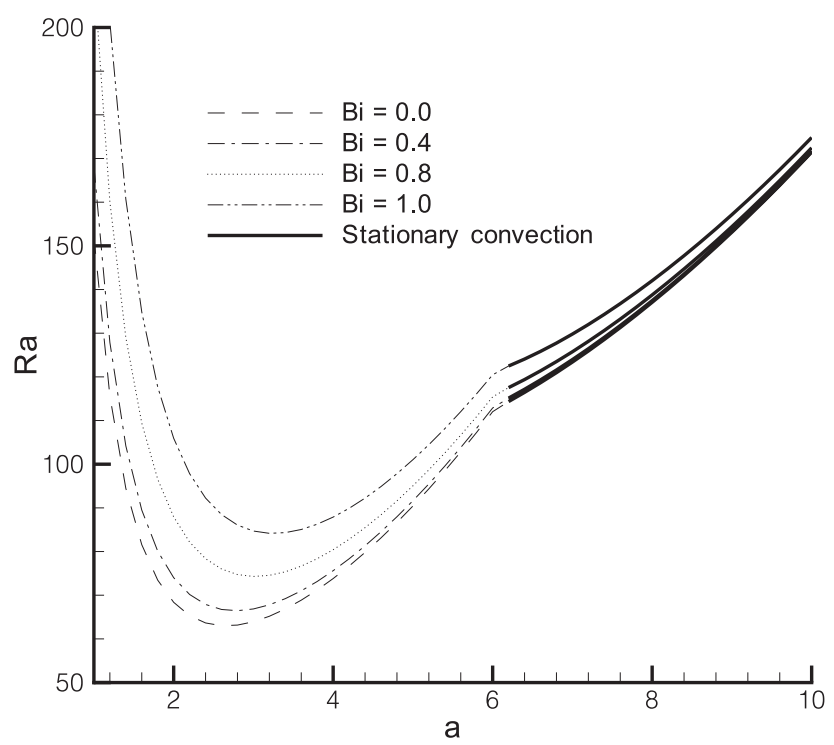

Fig. 7. Influence of the modified Biot number for $T a=5.0$, $D a=10^{-5}, S=1.0$ and $\chi=1.0$. Dashed curves represent overstable convection and the solid curve represents stationary convection.

very permeable porous medium behaves approximately like a fluid and Chandrasekhar [7] showed that overstable convection is restricted to very low values of the Prandtl number.

Finally, the effect of the modified Biot number on the stability curves is shown in Figure 7 . It is clear that, by decreasing $B i$ from $B i=1$ (purely conductive state) to $B i=0$ (perfectly adiabatically isolated boundary), one needs less energy input for temperature perturbations to develop, with, as consequence, a lower value of the critical temperature gradient. The difference is about $50 \%$ between the adiabatic case $(B i=0)$ and the purely conductive case $(B i=1)$. 


\section{Weakly nonlinear stability analysis}

\subsection{Nonlinear equations and amplitude equations}

The nonlinear evolutions of the vertical velocity, the vertical vorticity and the temperature are governed by the following dimensionless equations:

$$
\begin{aligned}
& \chi^{-1}\left(\partial_{t} \nabla^{2} w+\phi^{-1}\left[\nabla_{h}^{2} N(w)-\partial_{x z}^{2} N(u)-\partial_{y z}^{2} N(v)\right]\right)= \\
& R a \nabla_{h}^{2} T+\Lambda D a \nabla^{4} w-\nabla^{2} w-\sqrt{T a} \partial_{z} \zeta \\
& \chi^{-1} \partial_{t} \zeta+\chi^{-1} \phi^{-1}\left[\partial_{x} N(v)-\partial_{y} N(u)\right]= \\
& \quad \Lambda D a \nabla^{2} \zeta-\zeta+\sqrt{T a} \partial_{z} w \\
& S^{-1} \partial_{t} T+N(T)=\nabla^{2} T+w
\end{aligned}
$$

wherein $N(*)=\mathbf{u} \cdot \nabla *$.

The starting point of our nonlinear approach is the amplitude method which is described in details in $[11,12]$. This technique is based on the development of the solution in eigenmode series of the linear problem $\left(w_{P}, \zeta_{P}, T_{P}\right)$ :

$$
\left(\begin{array}{c}
w \\
\zeta \\
T
\end{array}\right)=\sum_{P} A_{P}(t)\left(\begin{array}{c}
w_{P} \\
\zeta_{P} \\
T_{P}
\end{array}\right)+\text { c.c. }
$$

index $P$ is written for $a, q$ and the $A_{P}(t)$ are the complex time-dependent amplitudes, c.c. stands for "complex conjugate". When development (33) is introduced in the nonlinear balance equations (30-32), the following amplitude equations are obtained for the amplitudes of the basic modes:

$$
\begin{aligned}
\frac{\mathrm{d} A_{P}}{\mathrm{~d} t}= & s_{P} A_{P}+\epsilon \sum_{Q} M_{P Q} A_{Q}+\sum_{Q, L} N_{P Q L} A_{Q} A_{L} \\
& +\sum_{Q, L, R} T_{P Q L R} A_{Q} A_{L} A_{R} .
\end{aligned}
$$

Definition of the basic modes and further details on the nonlinear technique are found in the appendix.

\subsection{Küppers-Lortz instability}

The presence of rotation in our system breaks the reflection symmetry, thereby allowing new instabilities to occur. We will focus on the so-called Küppers-Lortz instability discovered in 1969 in pure liquids [13]. This effect refers to the instability of a roll pattern with respect to a similar pattern but rotated through an angle $\alpha$ with respect to the original one. As the new pattern is also unstable to the same instability, the instability leads to a timedependent behavior and Küppers and Lortz showed that for sufficiently high rotation rates, this instability sets in immediately at the convection threshold. Numerous theoretical and numerical studies on Küppers-Lortz instability in pure fluids have been done [14-18] and a good review is found in [19]; however, there exists no equivalent work

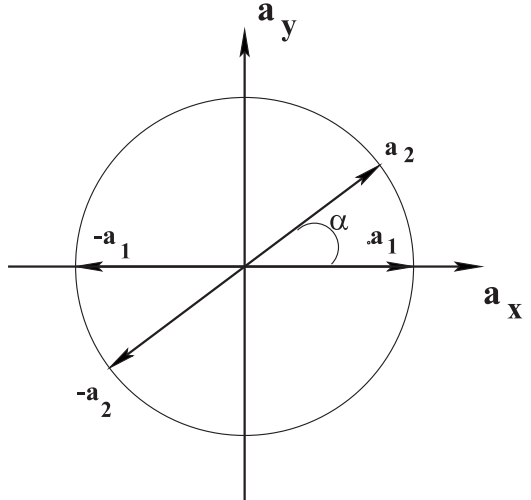

Fig. 8. Wave vectors $\mathbf{a}_{1}$ and $\mathbf{a}_{2}$ in the plane $\left(a_{x}, a_{y}\right)$.

for porous media and this lack is one of the motivations of the present section.

Since we are interested in the stability of a pattern of parallel rolls with respect to oblique roll-like disturbances, we formulate the nonlinear problem on a rhombic lattice whose angle $\alpha$ is to be specified. The lattice is thus defined by two wave vectors $\mathbf{a}_{1}$ and $\mathbf{a}_{2}$ (and their negatives) of magnitude $a_{c}$, satisfying $\mathbf{a}_{1} \cdot \mathbf{a}_{2}=a_{c}^{2} \cos \alpha$ (see Fig. 8) where a dot means a scalar product. We apply the technique described in the previous section to finally obtain the following two amplitude equations

$$
\begin{aligned}
& \tau \frac{\mathrm{d} A_{1}}{\mathrm{~d} t}=\epsilon A_{1}-\left(\Psi\left|A_{1}\right|^{2}+\Xi_{\alpha}\left|A_{2}\right|^{2}\right) A_{1}, \\
& \tau \frac{\mathrm{d} A_{2}}{\mathrm{~d} t}=\epsilon A_{2}-\left(\Psi\left|A_{2}\right|^{2}+\Xi_{\bar{\alpha}}\left|A_{1}\right|^{2}\right) A_{2},
\end{aligned}
$$

the quantities $A_{1}$ and $A_{2}$ denote the amplitudes of the modes $\mathbf{a}_{1}$ and $\mathbf{a}_{2}$, while the coefficients $\tau, \Psi, \Xi_{\alpha}$ and $\Xi_{\bar{\alpha}}$ are real. It is important to note that the presence of rotation breaks the symmetry $A_{1} \longleftrightarrow A_{2}$ characteristic of nonrotating systems and consequently, $\Xi_{\alpha}$ and $\Xi_{\bar{\alpha}}$ are not necessarily equals allowing Küppers-Lortz instability to occur. It is easy to check that rolls in the $\mathbf{a}_{1}$ direction lose their stability with respect to rolls in the $\mathbf{a}_{2}$ direction when $\Psi-\Xi_{\bar{\alpha}}$ passes through a zero and the onset of Küppers-Lortz instability is then located by using the following criterion [14]

$$
\min _{\alpha \in[0, \Pi]}\left[\Psi(\chi, T a)-\Xi_{\bar{\alpha}}(\chi, T a)\right]=0
$$

Equation (37) defines a critical Taylor number $T a_{c}$ and the corresponding angle $\alpha_{c}$ as a function of the filtration Prandtl number. In Section 3, it was stated that, in porous media, overstable convection is not restricted to particular values of the Prandtl number so that it is justified to consider the limiting case of an infinite Prandtl number, to be sure that the threshold will be stationary. Table 1 gives $\alpha_{c}$ and $T a_{c}$ as a function of $D a$. It is worth noticing that the results are not very sensitive for small values of $D a$ with a critical angle around $59^{\circ}$ and a critical Taylor number around 2. When $D a$ becomes much larger than one, the results coincide with the corresponding critical values for simple fluids, namely $\alpha_{c}=59^{\circ} .7$ and 
Table 1. Onset of Küppers-Lortz instability as a function of $D a$ for $\chi=\infty$.

\begin{tabular}{cll}
\hline$D a$ & $\alpha_{c}$ & $T a_{c}$ \\
\hline $10^{-8}$ & $58^{\circ} .6$ & 1.9 \\
$10^{-3}$ & $58^{\circ} .8$ & 2.0 \\
$10^{2}$ & $59^{\circ} .7$ & $3 \times 10^{7}$ \\
\hline
\end{tabular}

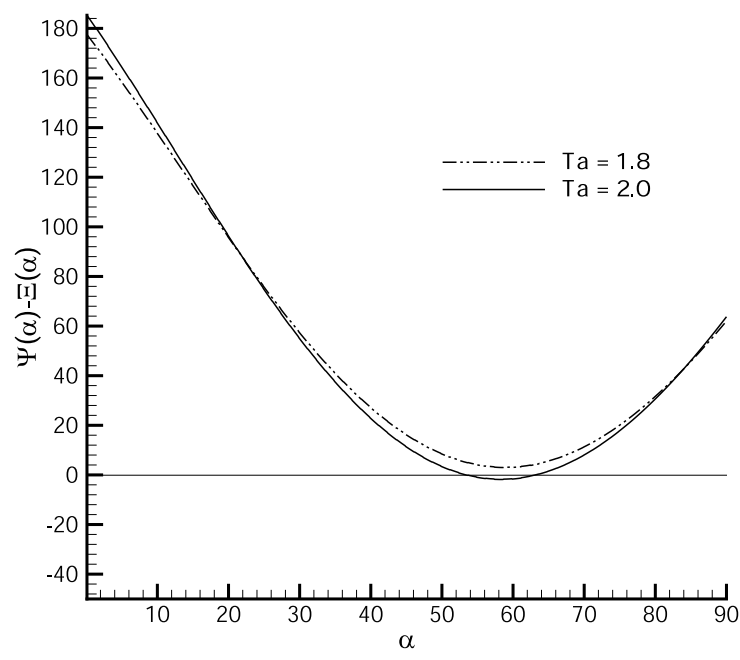

Fig. 9. $\Psi-\Xi_{\bar{\alpha}}$ as a function of $\alpha$ for $T a=1.8$ and $T a=2.0$.

$T a_{c}^{\text {fluid }}=T a_{c} D a^{-2}=3003$ [14]. In Figure 9 we represent $\Psi-\Xi_{\bar{\alpha}}$ as a function of $\alpha$ for Taylor numbers slightly below and above the critical value. We will not reproduce the nonlinear coefficients as they depend on the normalization used in the amplitude equations but it is worth noting that $\Psi$ is always positive. This confirms the result of Vadasz [2] that a pitchfork bifurcation occurs at the threshold of the stationary convection.

\section{Conclusions}

In the first part of this paper, a linear stability analysis is performed to study the onset of both stationary and overstable thermal convection in a rotating porous layer heated from below. The effect of rotation is restricted to the Coriolis term, neglecting thus the centrifugal force and heat transfer is described by Newton's cooling law. With respect to previous works, we have examined the effects resulting from the substitution of Darcy's by Brinkman's law.

The main results can be summarized as follows. First, it is observed that Brinkman's model gives qualitatively the same results as Darcy's one. Second, as already pointed out by Vadasz [2], overstable convection is not confined to particular values of the Prandtl number. Third, the overstable minimum in the neutral curves tends to disappear for large Darcy numbers.

In the second part of the work, special emphasis has been put on the onset of Küppers-Lortz instability for an infinite Prandtl number by means of a weakly nonlinear approach. We have derived the Ginzburg-Landau equations in a general way and considered the particular situation of two sets of rolls rotated by an angle $\alpha$. The critical angle $\alpha_{c}$ and the corresponding Taylor number are calculated. They are shown to behave nearly independently for small $D a$ 's and the single fluid case is recovered for large $D a$ 's.

In a work now in progress, we will study in more details the spatio-temporal patterns resulting from the KüppersLortz instability and compare our nonlinear results with direct numerical simulations based on the full 3D nonlinear equations.

This work was supported by ESA through the CIMEX-MAP and PRODEX contracts and by the European Union through ICOPAC project HRPN-CT-2000-00136. The authors thank Prof. E. Arquis and Prof. J.P. Caltagirone (MASTER Laboratory, ENSCPB, Bordeaux) as well as Prof. W. Pesch (University of Bayreuth) for stimulating discussions.

\section{Appendix}

In this appendix we present in detail the nonlinear method used in this paper and the way to obtain the amplitude equations (34). After introducing expansions (33) in equations (30-32), one projects them on the adjoint eigenfunctions of the linear problem. This amounts to multiply equation (30) by $w_{P}^{\star}$, the vertical velocity field of the adjoint eigenvalue problem, equation $(31)$ by $\zeta_{P}^{\star}$, the vertical vorticity field of the adjoint eigenvalue problem and finally the energy equation by $T_{P}^{\star}$, with $T_{P}^{\star}$ the temperature field of the adjoint eigenvalue problem. These three relations are added and integrated over the system volume. Making use of the bi-orthogonality relations between the solutions of the eigenvalue problem and its adjoint, together with the boundary conditions, we obtain the time evolution equations for the amplitudes in the following form:

$$
\frac{\mathrm{d} A_{P}}{\mathrm{~d} t}=s_{P} A_{P}+\epsilon \sum_{Q} M_{P Q} A_{Q}+\sum_{Q, L} N_{P Q L} A_{Q} A_{L} .
$$

In equation (38), the quantity $\epsilon$ stands for $\left(R a-R a_{c}\right) / R a_{c}$, i.e. the relative distance to the threshold and the matrices $M_{P Q}$ and $N_{P Q L}$ are given by:

$$
\begin{aligned}
M_{P Q}= & R a_{c} \frac{\left\langle w_{P}^{\star} \nabla_{h}^{2} \theta_{Q}\right\rangle}{\left\langle S^{-1} \theta_{P}^{\star} \theta_{P}+\chi^{-1}\left(w_{P}^{\star} \nabla^{2} w_{P}+\zeta_{P}^{\star} \zeta_{P}\right)\right\rangle} \\
N_{P Q L}= & -\Upsilon^{-1}\left(\left\langle\chi ^ { - 1 } \phi ^ { - 1 } \left[w _ { P } ^ { \star } \left(\nabla_{h}^{2} N_{Q}\left(w_{L}\right)-\partial_{x z}^{2} N_{Q}\left(u_{L}\right)\right.\right.\right.\right. \\
& \left.\left.-\partial_{y z}^{2} N_{Q}\left(v_{L}\right)\right)+\zeta_{P}^{\star}\left(\partial_{x} N_{Q}\left(v_{L}\right)-\partial_{y} N_{Q}\left(u_{L}\right)\right)\right] \\
& \left.\left.+\theta_{P}^{\star} N_{Q}\left(T_{L}\right)\right\rangle\right)
\end{aligned}
$$

where square brackets denote integration over the fluid volume and $N_{i}\left(x_{j}\right)$ is a simplified notation for $\mathbf{u}_{i} \cdot \nabla x_{j}$ $(i, j=Q, L)$ while $\Upsilon$ is given by

$$
\Upsilon=\left\langle S^{-1} \theta_{P}^{\star} \theta_{P}+\chi^{-1}\left(w_{P}^{\star} \nabla^{2} w_{P}+\zeta_{P}^{\star} \zeta_{P}\right)\right\rangle .
$$


Referring to a slaving principle $[20,21]$, we are able to reduce the infinite dimensional set of equations (38) to a finite number of ordinary differential equations for the amplitudes of the most unstable modes of convection. The procedure leading to this reduced system can be briefly summarized as follows. First, the infinite number of eigenmodes is split into two categories. The "basic" modes are the most unstable modes, with real part of the growth rates close to zero while the "slaved" or "stable" modes are quickly damped, due to strong negative values for $\operatorname{Re}\left(s_{P}\right)$. These slaved modes are present in the solution only as the quadratic response to the nonlinear growth of the basic modes above the threshold and their own dynamics can be neglected. For this reason, the corresponding time derivatives are set equal to zero in the evolution equations (38). This results in an algebraic relation between the basic and slaved amplitudes. When the amplitudes of the slaved modes are small with respect to the basic ones, the quadratic terms of these algebraic equations containing only damped modes can be neglected and the following expression of the slaved amplitudes in terms of the basic ones can easily be deduced

$$
A_{P_{s}}=-\frac{1}{s_{P_{s}}} \sum_{Q, L} N_{P_{s} Q L} A_{Q} A_{L}
$$

where the sub-index $s$ refers to a slaved mode. In the r.h.s. of (42), the indices $Q$ and $L$ denote the basic modes only. Note that relation (42), based on the smallness of $A_{P_{s}}$, is always correct close to the threshold where the amplitudes of the basic modes can be assumed to remain sufficiently small. Expressions (42) for the amplitudes of the slaved modes are then introduced in the evolution equations (38) for the basic modes. If terms of order higher than 3 are neglected, one obtains the following final "amplitude equations" for the amplitudes of the basic modes:

$$
\begin{aligned}
\frac{\mathrm{d} A_{P}}{\mathrm{~d} t}= & s_{P} A_{P}+\epsilon \sum_{Q} M_{P Q} A_{Q}+\sum_{Q, L} N_{P Q L} A_{Q} A_{L} \\
& +\sum_{Q, L, R} T_{P Q L R} A_{Q} A_{L} A_{R} .
\end{aligned}
$$

In this relation, all amplitudes and indices correspond to basic modes only and the definition of the matrix $T_{P Q R L}$ is easily deduced from the context.

\section{References}

1. D.A. Nield, Convection in Porous Media, 2nd edn. (Springer-Verlag, 1999)

2. P. Vadasz, Free convection in rotating porous media, in Transport Phenomena in Porous Media, edited by D.B. Ingham, I. Pop (Elsevier, Oxford, 1998), pp. 285-312

3. R. Friedrich, Z. Angew. Math. Mech. 63, T246 (1983)

4. H.C. Brinkman, Appl. Sci. Res. A 1, 27 (1947)

5. E. Palm, P.A. Tyvand, Z. Angew, Math. Phys. 35, 122 (1984)

6. P. Vadasz, J. Fluid Mech. 376, 351 (1998)

7. S. Chandrasekhar, Hydrodynamic and Hydromagnetic Stability (The Clarendon Press, Oxford, 1961)

8. H.P. Greenspan, The Theory of Rotating Fluids (Cambridge University Press, UK, 1968)

9. P. Vadasz, Transp. porous media 23, 155 (1996)

10. C. Canuto, M.Y. Hussaini, A. Quarteroni, T.A. Zang, Spectral Methods in Fluid Dynamics (Springer-Verlag, 1987)

11. P.M. Parmentier, V.C. Regnier, G. Lebon, J.C. Legros, Phys. Rev. E 54, 411 (1996)

12. P. Colinet, J.C. Legros, M.G. Velarde, Nonlinear Dynamics of Surface Tension Driven Instabilities (Wiley-VCH, 2001)

13. G. Küppers, D. Lortz, J. Fluid Mech. 35, 609 (1969)

14. T. Clune, E. Knobloch, Phys. Rev. E 47, 2536 (1993)

15. Y. Hu, W. Pesch, G. Ahlers, R.E. Ecke, Phys. Rev. E 58, $5821(1998)$

16. K.M.S. Bajaj, J. Liu, B. Naberhuis, G. Ahlers, Phys. Rev. Lett. 81, 806 (1998)

17. K.M.S. Bajaj, G. Ahlers, W. Pesch, Phys. Rev. E 65, 056309 (2002)

18. S.M Cox, P.C Matthews, J. Fluid Mech. 403, 153 (2000)

19. E. Knobloch, Int. J. Eng. Sci. 36, 1421 (1998)

20. P. Manneville, Dissipative Structures and Weak Turbulence, Perspectives in Physics (Academic Press, 1990)

21. H. Haken, Advance Synergetics (Springer-Verlag, Berlin, 1983) 